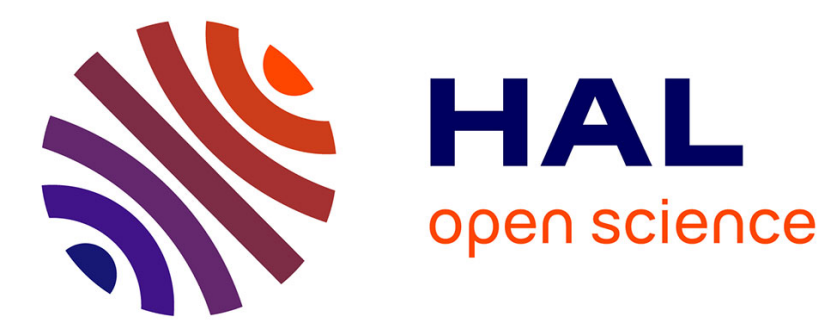

\title{
Fouilles au tumulus de Juniat, commune d'Asnières-sur-Blourde (Vienne) \\ Étienne Patte
}

\section{To cite this version:}

Étienne Patte. Fouilles au tumulus de Juniat, commune d'Asnières-sur-Blourde (Vienne). Gallia - Fouilles et monuments archéologiques en France métropolitaine, 1966, 24 (2), pp.231-234. 10.3406/galia.1966.2444 . hal-01934396

\section{HAL Id: hal-01934396 \\ https://hal.science/hal-01934396}

Submitted on 26 Feb 2020

HAL is a multi-disciplinary open access archive for the deposit and dissemination of scientific research documents, whether they are published or not. The documents may come from teaching and research institutions in France or abroad, or from public or private research centers.
L'archive ouverte pluridisciplinaire HAL, est destinée au dépôt et à la diffusion de documents scientifiques de niveau recherche, publiés ou non, émanant des établissements d'enseignement et de recherche français ou étrangers, des laboratoires publics ou privés.

\section{(이) $\$$}

Distributed under a Creative Commons Attribution - NonCommercial - NoDerivatives| 4.0 


\section{Fouilles au tumulus de Juniat, commune d'Asnières-sur-Blourde (Vienne)}

C'est au regretté commandant comte Henri de la Bouillerie que jai dû la connaissance de ce monument et la facilité de l'explorer. Juniat, Juniac sur la carte de Cassini, non mentionnć sur la carle d'ÉtatMajor au 1:80.000, comprend plusieurs maisons isolées situées au sud de la route d'Availleslimouzine a Luchapt, chef-lieu de commune le plus voisin (ces maisons écartées sont Ia Chantillone, La Porte, Le Logis, (irangeNeuve et Borliau). Le territoire d'Asnières touche a la fois à la Haute-Vienne et a la Charente. Encore en Poitou, il était en contact avec la province de la Basse-Marche et peu éloigné de l'Angoumois. Le tumulus se trouve au lieu-dit "Le Patural neuf ", parcelle 65) et empiète sur le lieu-dit "Lc Patural de la Motte", environ à 300 mètres au sud du carrefour de la route de Luchapt à AvaillesLimouzine et de l'allée empierrée allant au domaine de La Porte, du Logis et de la Maisonneuve, et légèrement à l'ouest de cette allée (fig. 1 et 2). Le contour, certainement altéré par les travaux agricoles, n'est pas régulier. Les diametres sont d'environ 34 mètres, la hauteur est de 1,65 à $1,70 \mathrm{~m}$. J'ai pratiqué deux tranchées perpendiculaires l'une à l'autre, suivant les directions n.-s. et e.-o. magnétiques.

La succession des couches, d'épaisscurs variables, était la suivante : couche stérile supérieure, bien que contenant parfois quelques charbons ; - terre intermédiaire ; -- couche très charbonneuse; - substrat avec couche fine supérieure, blanche.

A $1,80,1,60$ et $1,30 \mathrm{~m}$ au-dessous du sommet suivant les points, des charbons de bois nombreux par place indiquent des emplacements de foyers. A còté d'une des pierres isolées, la couche noire de cendres se dédoublait sur une longueur d'un peu plus de $30 \mathrm{~cm}$. La couche noire, surmontée, par endroit, de silex et d'argile rouge n'était pas plane. Dans le fond, des pierres el fragments de briques ou de tuiles, de tailles variables, étaient disposées, plus ou moins isolées et sans plan apparent. J'ai relevé leurs positions. Je note quelques dimensions de blocs : $25 \mathrm{~cm} \times 15$ $\times 7,5$ (en aplite rose); $30 \times 25 \times 8$ (fig. 3 à 6 ) .

I.e matériel recueilli comprend de nombreux débris de vases el de tuiles, ce qui n'est pas étonnant pour une époque d'intense production céramique où la coutume était de déposer près du mort quantité de fragments; ainsi que l'abbé Cochet l'écrivait il y a un peu plus de cent ans : "pas une créature ne descendit dans la terre sans un fragment de tuile ou un morceau de poterie..., les pauvres même, qui ne pouvaient trouver un vase dans leur répertoire culinaire, donnaient une simple tuile ou le tesson d'un vase». Les fouilles ont porté sur 17 mètres carrés et procuré 178 fragments de vases, soit à peu près 10 par mètre carré. Il ne s'est pas rencontré de tessons a des profondeurs moindres que les cendres. Presque tous les tessons étaient d'une terre avec grains de sable parfois grossiers.

Des morceaux recueillis au fond en 6 points différents ont permis de reconstituer un morceau de vase ayant $16 \mathrm{~cm}$ de diamètre environ à la base et 24 au moins à la panse; la pâte de teinte brique rose, avec quelques gros grains de sable, est bien cuite (épaisseur $6 \mathrm{~mm}$ à la panse ; 8 et 9 au fond). Un fragment vient d'un vase de $30 \mathrm{~cm}$ de diamètre environ. In morceau de pied creux, probablement, avec gabarit anguleux, est en pâte grise, devenue rouge brique sur les deux faces; il présente un léger vernis vitreux jaune 


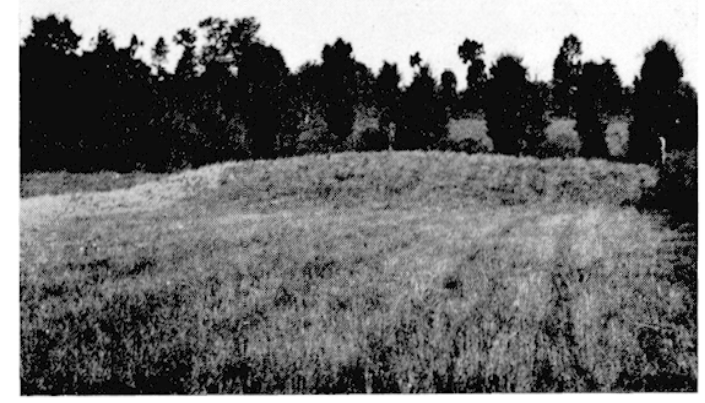

1. Le tumulus, pris du sud du sommet.

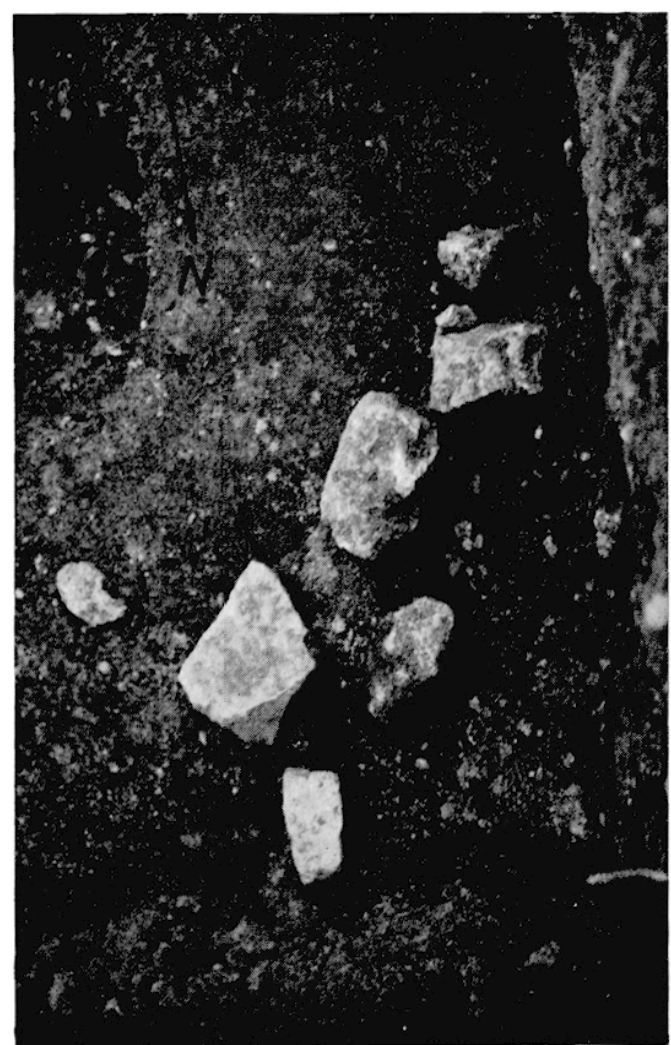

3. Photographie plongeante des blocs dégagés dans le coin nord-ouest de la fouille.

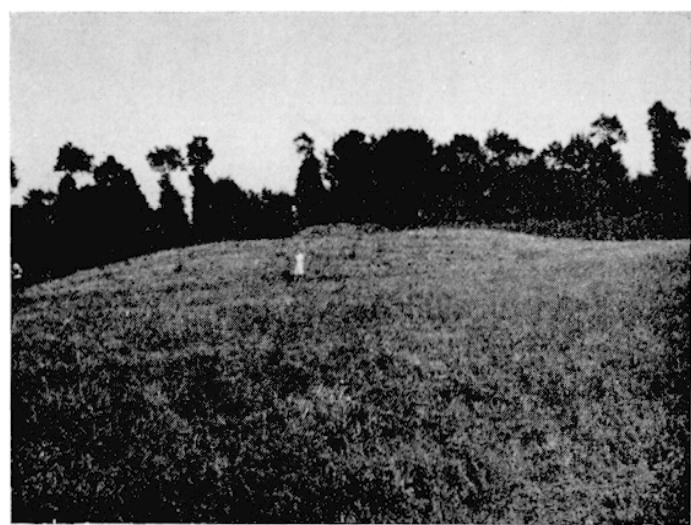

2. I.e tumulus, pris de l'ouest du sommet. (Les personnages indiquent respectivement le sommet et la base du tumulus.)

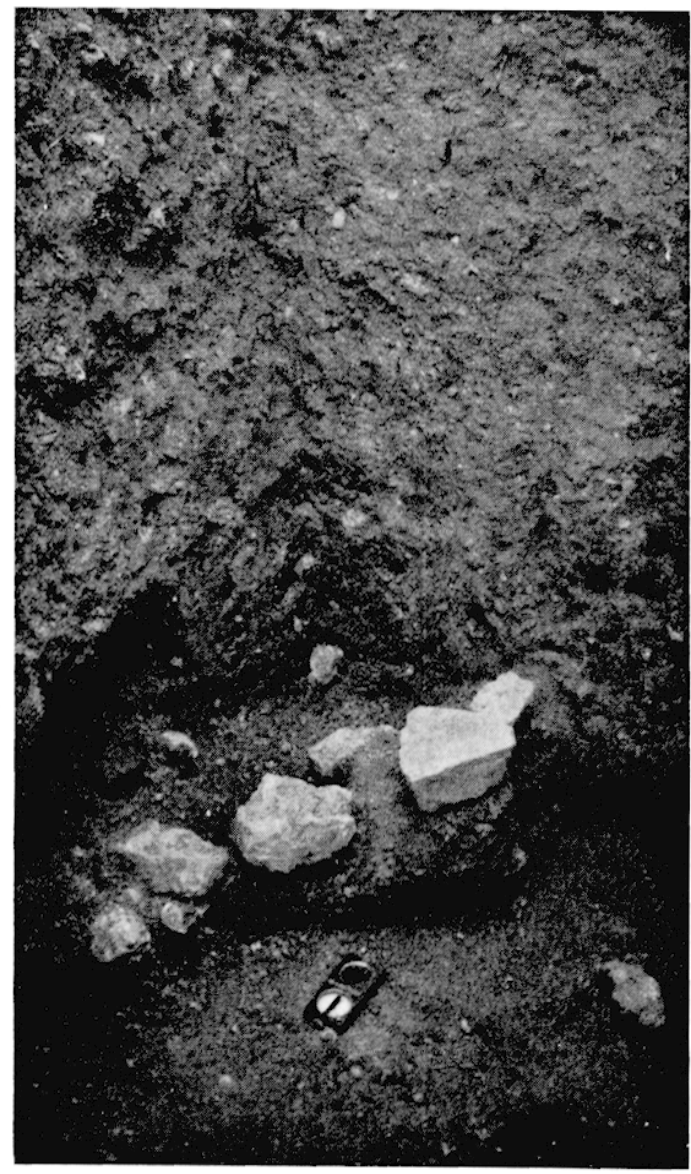

1. Photographie demi-plongeante, permetlant de voir : dans une niche pratiquée à l'ouest, un morceau de brique sombre et une pierre; dans l'angle nord-ouest, un autre bloc de faible taille. La boussole est orientée vers le nord magnétique et donne l'échelle (ayant $9,2 \times 18 \mathrm{~cm})$. 


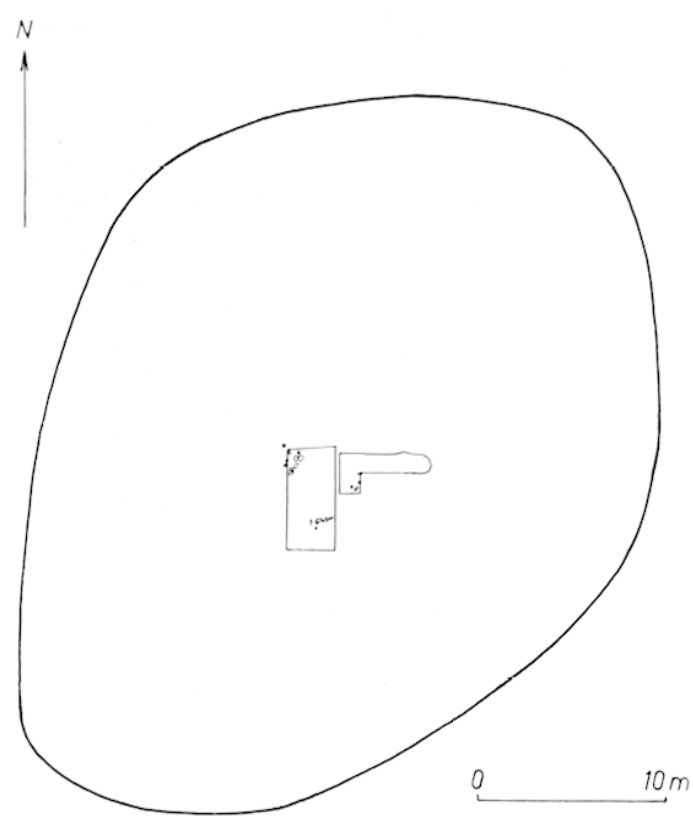

5. Plan du tumulus et des tranchees d'exploration.

verdâtre; le mème vernis se retrouve sur un fragment de pàte brique clair mais extérieurement grise; ces deux morceaux peuvent venir du mème pot. I in fond de vase de $13 \mathrm{~cm}$ de diametre, en lerre grise, épais de (i $\mathrm{mm}$ sur les parois, de $6, \overline{5} \mathrm{~mm}$ au fond, appartient peut-être au mème vase que deux bords avec levve tris saillante, à section anguleuse (épais de $4 \mathrm{~mm}$ au collet et 8 a la lìvre) et ayant $14 \mathrm{~cm}$ de diamètre. Un autre vase avait un fond a bord anguleux saillant. In autre encore, une anse en ruban, épaisse de $9 \mathrm{~mm}$, large de plus de 56 ; sa panse avait $3 \mathrm{~mm}$ d'épaisseur (couleur brique très claire). Enfin, un fragment d'anse à section ronde, épaisse de $18 \mathrm{~mm}$, est en pàte d'un rouge brique clair. Les autres débris ne peuvent donner aucune idée des formes ; les épaisseurs observées varient de :3 à $12 \mathrm{~mm}$ à la panse, de 4 a $14 \mathrm{~mm}$ au fond. Les épaisseurs les plus fréquentes vont de 4 a 6 .

Les couleurs sont tries variées :

$$
\text { pâte face externe face interne }
$$

blanche....... gris clair

blanche....... gris foncé

blanchâtre

brique claire

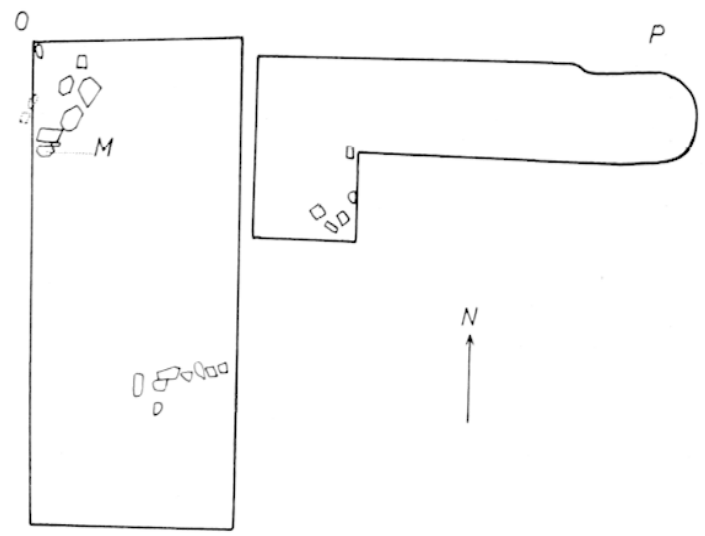

6. Détail des Iranchies, avec hocs, pierres on briques rencontris all fond. De 0 a $\mathrm{l}$, il y a $0,60 \mathrm{~m}$ de denivellation.

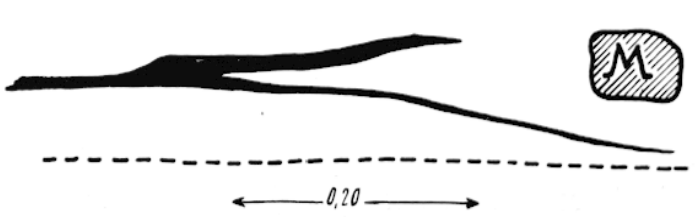

7. Coupe verticale montrant l'aspect des couches au niveau de la pierre M (roir le plan, fig. 6) ; la couche charbonneuse y est dídoublée. Les couches charbonneuses ont une forme conique. Le trait interrompu indique la base de la fouille.

blanchitre...... gris foncé

blanche............... rosée

blanche....... rosée

blanchàtre...... noire

très claire...... blanchâtre

uris............ blanchitre

gris noir

gris clair...... noire....... gris clair

gris

gris................ brique

jaune clair

brique........ gris

brique....... noir

rouge........ grise....... grise

brique claire

brique très claire

brique claire.... brique....... gris

noire......... noire....... noire

noire......... brique...... brique

noire......... brique....... noire

gris foncé...... gris foncé.... brique clair

gris.......... brique...... gris

brique claire.... gris sale 
gris clair verdâtre

noire......... gris clair..... rose

brique claire..... grise

brique jaunâtre

rose

La cuisson est presque toujours très bonne.

En outre, ont été recueillis 3 fragments de briques, 8 ou 9 de briques peu épaisses ou de tuiles, un fragment de tuile à rebords.

Les débris de fer sont peu nombreux (8) et méconnaissables. Il faut ajouter une pièce fourchue; les deux branches se rapprochent en formant un angle aigu; la forme rappelle ainsi le squelette d'un bec d'oiseau; l'ouverture était d'environ $12,5 \mathrm{~cm}$, la longueur de 17 . - In morceau de verre mince et vert est identique à celui des urnes cinéraires que l'on retrouve si fréquemment. - Diverses pierres avaient été apportées : silex, granites, aplites, etc. - Enfin, les débris d'os rencontrés m'ont rappelé ceux que j'ai pu voir aux abords du four crématoire d'Óswiecim (Auschwitz).

Ces observations rappellent les remarques de Martial Imbert ${ }^{1}$ sur les tumulus de la Haute-Vienne ; tous ceux qui ont été fouillés contiennent du fer, indiquent l'incinération et une population peu fortunée. L'incinération ne disparut en Lyonnaise qu'au ive ou $v^{e}$ siècle; elle était encore pratiquée au ${ }_{\text {III }}{ }^{\mathrm{e}}$ siècle, au moins en Bas-Limousin.

Ce tumulus se trouvait à moins d'un kilomètre de la voie romaine de Poitiers à Limoges ; celle-ci, d'après Lièvre, venant du n.-n.-o., passait à "Chez Touraud", près de Broux, puis obliquait vers Luchapt et le château de Montbron.

\section{Étienne Patte}

(1) M. IMBERT, Considérations générales sur les lumulus du Limousin, dans L'Homme préhistorique, 1904, p. 4. 